\title{
Morton's Neuroma
}

\author{
Arvind Puri
}

\begin{abstract}
Morton's neuroma is a common cause of forefoot pain. It most commonly affects middle-aged females and involves the third web space. A thorough history and examination should be adequate to diagnose with ultrasound (US) being the investigation of choice. Treatment usually involves conservative measures including analgesia; footwear and orthotic; local steroid injection; and in cases of failed nonoperative measures, open excision is performed.
\end{abstract}

Keywords: Foot, Neuropathy, Nonoperative, Pain, Surgical technique.

Journal of Foot and Ankle Surgery (Asia Pacific) (2020): 10.5005/jp-journals-10040-1122

\section{INTRODUCTION}

Morton's neuroma is a compressive neuropathy involving the interdigital nerves in the foot, commonest being the digital nerve in the third web space. It is still controversial if this is truly a "neuroma" or a degenerative neuropathy as the histopathology does not demonstrate typical features of a neuroma but that of perineural fibrosis and neural degeneration and demyelination. ${ }^{1}$

It is one of the more common causes of metatarsalgia and has been reported to have an incidence of $50.2 \%$ for men and $87.2 \%$ for women per 100,000 of patients presenting to primary care in the UK. ${ }^{2}$ There exists a female preponderance (4:1 female-to-male ratio) and usually presents in middle age. ${ }^{3}$ The condition is bilateral in $21 \%$ of cases with the third web space involved in most patients (66\%). The second web space accounts for $30 \%$ and the fourth in less than $2 \%$ of cases. ${ }^{4}$ It is rare to have multiple web spaces involved.

\section{Etiology}

Various theories have been proposed to explain this clinical entity. The most common is based on the anatomy of the digital nerves. Tibial nerve divides into a lateral and medial plantar branch. The medial branch divides to form the common digital nerve of the first, second, and the third web space as well as the digital nerve proper on the medial side of the hallux. The lateral contributes to the fourth web space as the common digital branch and the digital nerve proper on the lateral aspect of the little toe. There exists an anastomotic branch from the common digital nerve of the fourth web space to the nerve in the third web space. It has been reported to be present in $66.2 \%$ of cases. ${ }^{5}$ This results in the common digital nerve of the third web space being thicker and more prone to compression and trauma as the nerve traverses under the deep transverse metatarsal ligament. ${ }^{5-7}$ All the common digital nerves end up by bifurcating and supplying the adjacent toes (Fig. 1).

Limited space (bursal inflammation and thickening and the presence of ganglion or other space occupying lesions in the web space), trauma, and adjacent metatarsophalangeal (MTP) joint inflammation have all been implicated in the pathogenesis of Morton's neuroma. Its association with the usage of high heels and tight gastrocnemius is well-recognized. ${ }^{8}$

\section{Clinical Presentation}

Classic symptom experienced by the patients is pain in the web space on the plantar surface. This is usually described as
Cairns Hospital, Queensland, Australia

Corresponding Author: Arvind Puri, Cairns Hospital, Queensland, Australia, Phone: +61 439655363, e-mail: arvind.puri@health.qld. gov.au

How to cite this article: Puri A. Morton's Neuroma. J Foot Ankle Surg (Asia Pacific) 2020;7(2):46-49.

Source of support: Nil

Conflict of interest: None

burning, shooting, or cramp-like and associated with weightbearing. Sometimes, patients will complain of neuropathic pain with radiation to the toes. Pain is accentuated by tight-closed in shoes-patients typically described having to take shoes off and massage the forefoot to obtain relief. Bare feet weight-bearing on hard surfaces exacerbates the pain as sometimes reported by the patients.

The diagnosis of a Morton's neuroma rests on obtaining a good history and performing a methodical and thorough examination. ${ }^{9}$ In most patients, this will clinch the diagnosis with investigations

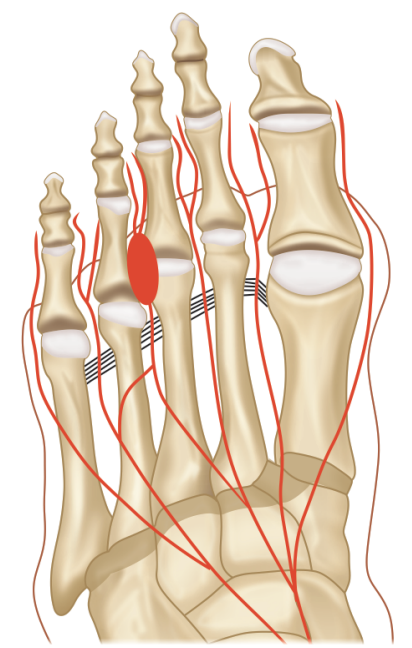

Fig. 1: Subdivision of plantar nerves. (Courtesy: Caprio FD, Meringolo R, Eddine MS. Morton's interdigital neuroma of the foot: A literature review. Foot and Ankle Surgery 2018;24:92-98) 
assisting in questionable cases or where the pathology may be other than Morton's neuroma.

\section{Clinical Examination}

The patient should have the lower limbs, below the knee, exposed to conduct a systematic and through examination.

It is crucial, even though the symptoms are focused on the forefoot, that the examination commences at the hindfoot and progress toward the forefoot to extract information that confirms the presence of Morton's neuroma or directs toward another causative pathology.

\section{Look}

Inspection of the hindfoot alignment (Valgus or Varus), the height of medial longitudinal arch (normal, high or planus), forefoot in relation to hindfoot (abducted, adducted, pronated, supinated), the presence of toes abnormalities (hallux valgus, hammer toe, claw toe, increased gaping between less toes), and the presence of any plantar callosities provide essential information.

Any swelling/s, previous surgical or traumatic scars, and appearance of skin conditions are important pick-ups.

Gait: observe the gait of the patient paying special attention to the three rockers - heel strike, mid-stance, and toe off. The inability to weight-bear on a plantigrade foot provides a clue to where the pathology may be.

\section{Feel}

Palpation to delineate the specific tender area is crucial. Intermetatarsal space tenderness on the plantar surface is typical of Morton's neuroma. Metatarsophalangeal joint or tenderness localized to the metatarsal heads favors another pathologyplantar plate tear, hammer/claw toe, MTP joint arthritis, avascular necrosis (AVN) metatarsal head, or an instability issue.

\section{Move}

Sequential examination of the range of joint motion, passive and active, from the hindfoot to the toes is performed next.

Comparison is made with the opposite, if normal, side. Equinus at the ankle and hypermobile first ray will contribute to excessive forefoot pressure and consequent metatarsalgia.

Stiffness at the TMT articulation may also cause uneven forefoot weight distribution.

\section{Special Tests}

Mulder's sign ${ }^{10}$ or click is considered pathognomonic for Morton's neuroma and has a sensitivity of $94-98 \%$. $^{11}$

This sign is elicited by squeezing the metatarsals together with one hand while grasping the forefoot from the dorsum. The thumb on the other hand is used to apply pressure over the concerned intermetatarsal space from the plantar aspect. A painful and palpable click is considered positive (Fig. 2).

Silverskold test for gastrocnemius tightness is important, if positive, as it can be an additional contributing cause of metatarsalgia.

Divergence of adjacent toes at the symptomatic web space is also considered to be a sign of Morton's neuroma. The deviation results from a neuroma that is large enough to push the metatarsal heads apart.

The examination should include a through neurovascular assessment of the foot.

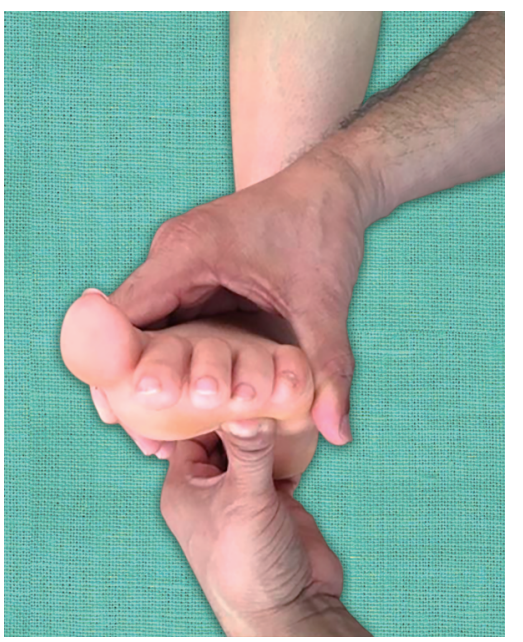

Fig. 2: Technique for eliciting Mulder's sign or click

\section{INVESTIGATIONS}

In our setting, most patients present with an X-ray and US and, in some cases, a magnetic resonance imaging (MRI). The consultation should always commence with history followed by examination, and only then, attention should be given to the investigations.

A weight-bearing X-ray is useful to rule out MTP arthritis, AVN metatarsal head, relative lengths of metatarsals and TMT arthritis.

Ultrasound is a dynamic study and the investigation of choice which can confirm the presence of a neuroma or other spaceoccupying lesion as well as bursal involvement. It has a $90 \%$ sensitivity and $88 \%$ specificity. ${ }^{12}$ No correlation has been found between the size of the neuroma and the presence of symptoms. It, however, has the additional advantage of injecting local anesthetic to provide diagnostic evidence. ${ }^{13}$

Magnetic resonance imagings are $93 \%$ sensitive but have a poor specificity of just $68 \% .^{12}$

\section{Treatment}

\section{Conservative Management}

Conservative management is the mainstay of initial treatment. Once the diagnosis of Morton's neuroma is established, the following nonoperative options can be utilized.

\section{Analgesia}

Appropriate and regular analgesia should be prescribed.

\section{Physiotherapy}

This should include gastrocnemius stretching and intrinsic exercises.

\section{Appropriate Footwear and Orthosis}

Wide forefoot-fitting shoes with metatarsal head offloading orthosis.

\section{Activity Modification}

Desist impact activities_running and jumping.

\section{Steroid and Local Injections}

This has been conducted under US guidance with an injection of a combination of steroid and local anesthesia beneath the deep transverse intermetatarsal ligament and has been shown to be effective in $30 \%$ of patients. ${ }^{14-16}$ 
Injections can also delay the need for surgical intervention which has been reported to be performed in $70 \%$ of patients after injection. ${ }^{17}$ The beneficial effects have been reported to last up to 6-12 months. ${ }^{18}$

The other nonoperative interventions are radiofrequency ablation, ${ }^{19}$ alcohol injection, and Botulinum toxin $\mathrm{A}$.

Cryotherapy, extracorporeal shockwave therapy, and laser therapy have all shown mixed, temporary results.

\section{Surgical Treatment}

Surgical intervention is considered when conservative and infiltrative means have failed or symptoms have recurred.

Some studies have suggested that surgical intervention should be offered at the outset if symptoms are typical of Morton's neuroma and have been present for a year.

Results of surgery have been superior if the neuroma measures more than $5 \mathrm{~mm}$ on US scan.

The available surgical options are as follows:

- Neurectomy and decompression

- Decompression with/without Weil's osteotomy

My preferred surgical option is neurectomy and decompression through a dorsal approach. This is the more popular and practiced approach. This allows one to decompress and perform neurectomy. I reserve the plantar approach for recurrent Morton's or Stump neuroma.

\section{Technique}

Under the general or regional block, patient is placed supine with a sand bag under the ipsilateral hip. Thigh tourniquet is inflated after prophylactic antibiotic is given.

Incision extents from the involved web space to proximal and to the metatarsal heads.

Sharp and blunt dissection is performed until the transverse intermetatarsal ligament is exposed. If digital pressure is applied on the plantar surface of the web space, the neuroma can be visualized.

Using lamina spreaders, the adjacent metatarsal heads can be retracted and the ligament is placed at stretch. A McDonalds or Freer elevator can be inserted under the ligament, and then, it can be divided safely.

This exposes the neurovascular bundle, and the neuroma is clearly visualized. Meticulous dissection (loupes can be helpful) should be performed to isolate the common digital nerve proximally, the neuroma past the divided ligament, and the bifurcation of the nerve in the web space. Avoid excising fat from the plantar aspect.

A sharp division of the nerve $3-4 \mathrm{~cm}$ proximally should be carried out. This allows the stump to be buried in the muscular tissue. Similarly, the two digital branches should be divided, and the specimen has been sent for histopathological examination.

I then deflate the tourniquet, control bleeding, and close the wound with absorbable suture to deep tissue and skin.

Wound is dressed with an occlusive dressing followed by wool and crepe compression.

Postoperative regime is rest and elevation for 10 days with short walks and heel weight-bearing in a Darco shoe.

Wound is inspected at the two-week mark, and if healed, patient can increase activity in a graduated fashion.

At six weeks, patient can return to all activities except for high impact.

The surgical scar can be massaged with moisturizing cream.

\section{Discussion}

Morton's neuroma is a common clinical entity and can be diagnosed by obtaining a detailed history and performing a thorough clinical examination. There is a very limited indication for employing radiological investigations to confirm the diagnosis.

The most common web space involved is the third web space followed by the second.

The typical symptoms reported by the patient are pain on the plantar side of the web space in-between the adjacent metatarsal heads. Tight-fitting shoes accentuate the discomfort, and patients often describe the "presence of a pebble" in the shoe on weightbearing.

Examination should be methodical and proceed from hind to fore foot with special consideration to performing the Silverskold test and eliciting Mulder's click. Other potential causes for metatarsalgia should be excluded with examination.

Weight-bearing foot X-rays should be done to rule out other bony or joint pathologies or deformities which may be the cause of metatarsalgia or contributing to the symptoms of neuroma as well.

Ultrasound is useful in confirming the presence and measuring the size of the neuroma. It can confirm the diagnoses by injecting local anesthetic plantar to the transverse metatarsal ligament in the concerned web space. If the patient reports resolution of symptoms, it clinches the diagnoses in questionable cases. Ultrasound is important for conducting the infiltrative mode of treatment whether using steroids, local anesthesia (LA), alcohol, or Botulinum toxin A. It has also been used to accurately locate the neuroma while using radiofrequency ablation.

The first line of treatment should be conservative and infiltrative. A systematic review of nonsurgical management concluded that corticosteroid injections manipulate with the strongest evidence for pain reduction. ${ }^{20}$

Another systematic review concluded that operative treatment showed better results with $70-80 \%$ success rate. ${ }^{21}$ This, however, is tempered by the findings that the complication rate of surgery was $21 \%$. The recurrence rate, on the other hand, was higher with both conservative and infiltrative modes-up to $14 \% .^{22}$

Recurrence after surgery is usually a stump neuroma which is difficult to treat and has been shown to have poorer outcome.

\section{References}

1. Graham CE, Graham DM. Morton's neuroma: a microscopic evaluation. Foot Ankle 1981;5(3):150-153. DOI: 10.1177/107110078400500308.

2. Latinovic R, Gulliford MC, Hughes RAC. Incidence of common compressive neuropathies in primary care. J Neurol Neurosurg Psychiatry 2006;77(2):263-265. DOI: 10.1136/jnnp.2005.066696.

3. Bradley N, Miller WA, Evans JP. Plantar neuroma: analysis of results following surgical excision in 145 patients. South Med 1976;69(7):853854. DOI: 10.1097/00007611-197607000-00014.

4. Thomson FM, Deland JT.Occurrence of two interdigital neuromas in one foot. Foot Ankle 1993;14(1):15-17. DOI: 10.1177/107110079301400103.

5. Frank PW, Bakkum BW, Darby SA. The communicating branch of the lateral plantar nerve: a descriptive anatomic study. Clin Anat 1996;9(4):237-243. DOI: 10.1002/(SICI)1098-2353(1996)9:43.0.CO;2-B.

6. Jones JR, Klenerman L. A study of the communicating branch between the medial and lateral plantar nerves. Foot Ankle 1984;4(6):313-315. DOI: $10.1177 / 107110078400400608$.

7. Diez EM, Mas SM, Pi JF, et al. Comparative results of two different techniques in the treatment of the Morton's interdigital neuroma. Foot 1999;9(3):134-137. DOI: 10.1054/foot.1999.0543. 
8. Bennett GL, Graham CE, Mauldin DM. Morton's interdigital neuroma: a comprehensive treatment protocol. Foot Ankle Int 1995;16(12):760763. DOI: $10.1177 / 107110079501601204$.

9. Couglin MJ. Common causes of pain in the forefoot in adults. J Bone Joint Surg Br 2000;82(6):781-790. DOI: 10.1302/0301620X.82B6.0820781.

10. Mulder JD. The causative mechanism in Morton's metatarsalgia. J Bone Joint Surg Br 1951;33(1):94-95. DOI: 10.1302/0301-620X.33B1.94.

11. Claassan $L$, Bock K, Erringer $M$, et al. Role of MRI in detection of Morton's neuroma. Foot Ankle Int 2014;35(10):1002-1005. DOI: 10.1177/1071100714540888.

12. Mahadevan $D$, Venkatesan $M$, Bhatt $R$, et al. Diagnostic accuracy of clinical tests for Morton's neuroma Compared with ultrasonography. J Foot Ankle Surg 2015;54(4):549-553. DOI: 10.1053/j.jfas.2014.09.021.

13. Pastides $\mathrm{P}$, El-Sallakh S, Charalambides C. Morton's neuroma: a clinical versus radiological diagnosis. Foot ankle Surg 2012;18(1):22-24. DOI: 10.1016/j.fas.2011.01.007.

14. Schon L. An injection of corticosteroid plus anaesthetic was more effective than anaesthetic alone for Morton's neuroma. J Bone Joint Surg Am 2014;96(4):334. DOI: 10.2106/JBJS.9604.ebo028.

15. Rasmussen MR, Kitaoka HB, Patzer GL. Non operative treatment of plantar interdigital neuroma with single corticosteroid injection. Clin Orthop Relat Res 1996;326:188-193. DOI: 10.1097/00003086199605000-00022.
16. Markovic M, Crichton K, Read JW, et al. Efectiveness of ultrasoundguided corticosteroid injection in the treatment of Morton's neuroma. Foot Ankle Int 2008;29(5):483-487. DOI: 10.3113/FAI.2008.0483.

17. Di Caprio F, Meringolo R, Eddine MS, et al. Morton's interdigital neuroma of the foot: a literature review. Foot ankle Surg 2017;24(2): 92-98. DOI: 10.1016/j.fas.2017.01.007.

18. Nakki D, Haddad BZ, Majmood Z, et al. Efficacy of corticosteroid injection versus size of plantar interdigital neuroma. Foot Ankle Int 2012;33(9):722-726. DOI: 10.3113/FA.2012.0722.

19. Cuter GS, Chua YP, Connell DA, et al. Ultrasound-guided radiofrequency ablation in the management of interdigital (Morton's) neuroma. Skeletal Radial 2013;42(1):107-111. DOI: 10.1007/s00256-0121527-x.

20. Mattews BG, Hurn SE, Harding MP, et al. The effectiveness of nonsurgical interventions for common plantar digital compressive neuropathy (Morton's neuroma): a systematic review and metaanalysis. J Foot Ankle Res 2019;12(1):12. DOI: 10.1186/s13047-0190320-7.

21. Kasparek M, Schneider W. Surgical treatment of Morton's neuroma: clinical results after open excision. Int Orthop 2013;37(9):1857-1861. DOI: 10.1007/s00264-013-2002-6.

22. Valisena S, Petri GJ, Ferrero A. Treatment of Morton's neuroma: a systematic review. Foot and Ankle Surgery 2018;24(4):271-281. DOI: 10.1016/j.fas.2017.03.010. 